\title{
RESEARCH
}

Open Access

\section{Single level anterior cervical discectomy and fusion versus dynamic cervical implant: clinical and radiological outcome}

\author{
Omar El Farouk Ahmed ${ }^{*}$ (iD and Ahmed Galal
}

\begin{abstract}
Background: Although anterior cervical discectomy and fusion (ACDF) is considered a gold standard approach for surgical management of cervical disc herniation syndromes, the use of dynamic cervical implant (DCl) provided a novel technique that aims at reconstruction of the anterior column while facilitating controlled neck motion and reducing stress across the facet joints.
\end{abstract}

Aim of the work: The objective of this study is to evaluate the clinical and radiological outcome of the DCl surgery in comparison to that achieved with ACDF using a conventional polyethylethylketone (PEEK) cage.

Materials and methods: This is a retrospective comparative study of 30 patients, with single level cervical degenerative disc disease (DDD), managed by the authors, either by DCI $(n=15)$ or ACDF $(n=15)$. Clinical and radiologic outcomes were assessed at 1,3, and 12 months postoperatively. Clinical scoring systems included the Visual Analog Scale for Neck (VAS-N) and Arm (VAS-A), the Neck Disability Index score (NDI), as well as the evaluation of incidence of complications and neurological deterioration postoperatively. Radiographic evaluation included the assessment of postoperative cervical implant fusion, as well as evaluating the incidence of implant migration. Study duration was for two years from October 2016 to October 2018.

Results: Both the ACDF and DCl groups showed significant clinical improvement at 12 months postoperatively regarding the clinical outcome including VAS-A, VAS- $N$, and NDI values $(P=0.001)$, while there was no significant difference on comparing between the two groups as regard the VAS-N, the VAS-A, and the NDI at 1,3 and 12 months after surgery. The ACDF group however showed better rate of implant fusion at 12 months postoperatively in contrast to the DCl group ( $80 \%$ and $26,7 \%$, respectively) also, the ACDF group showed a lower rate of implant subsidence at 12 months after surgery $(P=0.002)$. Besides, the incidence of implant migration was relatively high in the DCl group (20\%).

Conclusion: The clinical results for $\mathrm{DCl}$ arthroplasty for the management of single-level cervical DDD are equivalent to those for ACDF; however, though providing an immediate dynamic stability, DCI is associated with a low fusion rate, higher rates of implant subsidence, and relatively high implant migration rate. Larger series and further studies should be considered with longer follow-up periods giving special attention to these issues.

Keywords: Dynamic cervical implant, DCl, Anterior cervical discectomy and fusion, Cervical disc disease, Adjacent segment disease

\footnotetext{
* Correspondence: omarelfarouk@hotmail.com

Department of Neurosurgery, Faculty of Medicine, Ain Shams University, Cairo, Egypt
} 


\section{Introduction}

Although anterior cervical discectomy and fusion (ACDF) using a PEEK cage is considered the gold standard measure for the surgical management of cervical degenerative disc disease (DDD [6, 12];) ; some studies suggested that the rigid fixation maybe associated with increased motion and increased intradiscal pressure in the untreated levels adjacent to fused levels and subsequently may predispose to an increased risk of adjacent segment disease [1].

New treatment approaches have been developed searching for the optimal interface mechanics between the device and adjacent bony endplates $[2,8]$ to restore or preserve the mobility of the involved segment. Dynamic cervical implant (DCI) introduced in the past decade is a U-shaped titanium self-fixing dynamic spacer that stabilizes the involved segment while maintaining a motion-preserving strategy as it fits the concave surfaces of the involved segment superior and inferior end plates via its sharp serrations (teeth) on the upper and lower ends to reduce the potential possibility of implant migration [9].

DCI theoretically provides controlled flexion-extension motion, the main subaxial spine motion, thus allowing the spine to be functionally dynamic, while limiting axial rotation and lateral bending, a motion which significantly contributes to facet forces, besides its shock absorption potential, to prevent transfer of stresses to adjacent levels, and consequently reduce the possibility of adjacent segment degeneration [10]. Recently published studies evaluated the safety and efficiency of DCIs in the management of single-segment cervical DDD [7, 15].

The objective of this study is to compare clinical and radiological outcome from DCI surgery with that achieved with ACDF using a PEEK cage.

\section{Material and methods}

This is a retrospective comparative study on 30 patients with single-level cervical disc herniation, conducted in the period between October 2016 to October 2018.

The patients were divided according to surgical approach into two groups, the ACDF group (15 patients) and the DCI group (15 patients) who were operated all through anterior approach for cervical discectomy surgery followed by implant insertion. Patients were followed up for 1, 3, 12 months. This study occurred at Ain Shams University Hospitals.

\section{Patient selection}

Inclusion criteria

Patients must have the following criteria:

1. Adult individuals aged over 18 years old
2. Patients with a single-level cervical disc prolapse between C3 and C7 presented with radicular symptoms and neck pain without any motor deficit

3. Radiological results confirming the diagnosis including plain X-ray (PXR; anteroposterior (A-P), lateral views, and dynamic views) and magnetic resonance imaging (MRI)

4. Failed conservative management for at least 12 weeks

\section{Exclusion criteria}

Patients must not have the following:

1. Patients with multiple-level cervical disc prolapse

2. Patients with recurrent cervical disc prolapse (same or new level)

3. Marked cervical instability (i.e., higher than 3-mm translation)

4. Patients with myelopathic symptoms and/or signs (to limit the possible variables in the study results)

5. The presence of other cervical spine pathologies such as trauma, tumor, infection, or deformity

6. Posterior compressive cervical pathology

\section{Preoperative data}

Clinical evaluation

This will include the following:

- Patients' demographics (age, sex)

- Relevant past medical and surgical history

- Full general and history and neurological examination

- Clinical presentation and its duration including assessment of the visual analogue scale (VAS) for both neck and arm (VAS-N and VAS-A, respectively) [4]

- Neck disability index (NDI) [14]

\section{Radiological investigation}

Plain radiographs The following are the anteroposterior, lateral, and dynamic views to evaluate:

- Anatomy of the cervical spines including evaluation of the facet joints; presence of degenerative changes; neural foraminal dimensions, and bone density

- Measurement of preoperative disc height at the surgical segment to assess stability of the spine on dynamic views.

MRI Magnetic resonance imaging (MRI) of the cervical spine without Gadolinium enhancement allowing anatomical evaluation of the spine and spinal canal, nerve roots, and spinal ligaments complex. 


\section{Surgical technique}

Following general anaesthesia and endotracheal intubation, all patients were positioned supine with head in neutral position. Skin incision was performed on the right side of the anterior aspect of the neck according to the operated level.

All had microscopic anterior cervical discectomy and decompression of the neural canal and neural foraminae.

The end plates were carefully cleaned for any residual disc material and any posterior bony osteophytes were removed using Kerrison Rongeurs and/or a drill then the posterior longitudinal ligament was resected.

Our patients were classified into two groups according to the surgical procedure, each consisted of 15 patients.

The first group had a conventional PEEK cage ( Medtronic), while the second group were given a DCI (Paradigm Spine). The determination of the implant size was done by cage trials and evaluation through intraoperative lateral radiography with special attention to maximize the end plate-implant contact. The implant was then inserted under slight distraction of the segment using CASPAR vertebral distractors then compression was applied to fixate the implant teeth/serrations of the implant against the bony end plates.

A lateral fluoroscopic image was obtained to ensure safe and proper positioning of the implant.

Finally, closure was performed in a routine fashion over a suction drain. All patients received prophylactic antibiotics perioperatively and were requested to start ambulation on the next day using a hard neck collar for a couple of months. The drains were removed at $24 \mathrm{~h}$.

Intraoperative and postoperative data including intraoperative blood loss, operative time, the incidence of postoperative neurological deficit, and the hospital stay were recorded.

All surgeons accounting for performing the two procedures were efficient with the same skill level.

\section{Evaluation follow up Clinical outcome}

Assessment of the patients' neurological outcome, for the two groups, through evaluation of the post-operative VAS scores for neck and arm pain as well as the NDI scores at 1, 3, and 12 months postoperatively in comparison to preoperative scores.

\section{Radiological outcome}

Radiological outcome was assessed by plain X-ray (A-P, lateral views) on the first postoperative day, then subsequent radiographs were done at 1,3 , and 12 months after surgery.

Radiological evaluation for both groups included the implant fusion rate, incidence of subsidence, assessment of Cobb's angle and the cervical lordotic curve, and the identification of possible implant migration defined as an A-P or lateral change of the implant position in comparison with the radiograph obtained on the first postoperative day. Whereas fusion was described as a less than 1-mm motion between the tips of the spinous processes in dynamic radiographs and/or the presence of bridging bony trabeculae.

\section{Statistical analysis}

Continuous variables are expressed as mean and standard deviation (SD). Categorical variables are expressed as frequencies and percents. Numerical variables were compared between the two study groups using student $t$ test. Chi square and Fisher's exact test were used to compare categorical variables. Repeated measure analysis of variance $(A N O V A)$ was used to compare means of clinical outcome measurements that is made several times. A significance level of $P<0.05$ was used in all tests. All statistical procedures were carried out using Statistical Package for the Social Sciences (SPSS) version 20 for Windows (SPSS Inc, Chicago, IL, USA).

\section{Results}

All the patients' preoperative data was studied and analyzed. There were 20 (67\%) males and 10 (33\%) female patients. Their age ranged from 32 to 60 years, with a mean age of $47.6( \pm 8.09 \mathrm{SD})$ years in the ACDF group, and a mean age of $46.6( \pm 8.79 \mathrm{SD})$ years in the DCI group.

In ACDF subjects, the pathological level was $\mathrm{C} 3-4$ in a single $(6.7 \%)$ patient, C4-5 in $3(20 \%)$ patients, C5-6 in 7 (46.7\%) patients, and C6-7 in 4 (26.7\%) patients in contrast to $1(6.7 \%), 4(26.7 \%), 7$ (46.7\%), and $3(20 \%)$ patients in the DCI group respectively.

The left side was the pathological side in 7 (46.7\%) patients and the right side accounted for 5 (33.3\%) patients in the ACDF group, while the remaining $3(20 \%)$ patients were presented with bilateral symptoms. In the DCI group, the left side was involved in 8 (53.3\%) patients, the right side in $5(33.3 \%)$ patients, and bilateral complaint was noted in $2(13.4 \%)$ cases.

There was no statistically significant difference found between both groups regarding demographic data of the studied patients (Table 1).

\section{ACDF group}

Both VAS-N and VAS-A expressed significant postoperative improvement following ACDF surgery. Considering VAS- $\mathrm{N}$ evaluation, the mean values for the preoperative assessment, at the first, third, and twelfth months follow-up showed a statistically highly significant values on comparing between the preoperative and the postoperative results throughout the follow-up intervals $(P=$ 0.001 ), a highly significant value on comparing the first 
Table 1 Demographic data of the studied patients

\begin{tabular}{|c|c|c|c|c|c|c|}
\hline & \multicolumn{4}{|l|}{ Group } & \multirow[t]{3}{*}{ P } & \multirow[t]{3}{*}{ Significance } \\
\hline & \multicolumn{2}{|l|}{ ACDF } & \multicolumn{2}{|l|}{$\mathrm{DCl}$} & & \\
\hline & Mean & $\pm \mathrm{SD}$ & Mean & $\pm \mathrm{SD}$ & & \\
\hline Age & 47.60 & 8.09 & 46.60 & 8.79 & $0.748 \neq$ & NS \\
\hline Duration (months) & 10.40 & 4.47 & 10.93 & 9.17 & $0.841 \neq$ & NS \\
\hline \multicolumn{7}{|l|}{ Sex } \\
\hline Male & 9 & $60.0 \%$ & 11 & $73.3 \%$ & $0.439^{*}$ & NS \\
\hline Female & 6 & $40.0 \%$ & 4 & $26.7 \%$ & & \\
\hline \multicolumn{7}{|l|}{ Brachialgia } \\
\hline Right & 5 & $33.3 \%$ & 5 & $33.3 \%$ & $1.0^{* *}$ & NS \\
\hline Left & 7 & $46.7 \%$ & 8 & $53.3 \%$ & & \\
\hline Bilateral & 3 & $20.0 \%$ & 2 & $13.3 \%$ & & \\
\hline \multicolumn{7}{|l|}{ Numbness } \\
\hline Negative & 3 & $20.0 \%$ & 8 & $53.3 \%$ & $0.058^{*}$ & NS \\
\hline Positive & 12 & $80.0 \%$ & 7 & $46.7 \%$ & & \\
\hline \multicolumn{7}{|l|}{ Level } \\
\hline 3,4 & 1 & $6.7 \%$ & 1 & $6.7 \%$ & $1.0^{* *}$ & NS \\
\hline 4,5 & 3 & $20.0 \%$ & 4 & $26.7 \%$ & & \\
\hline 5,6 & 7 & $46.7 \%$ & 7 & $46.7 \%$ & & \\
\hline 6,7 & 4 & $26.7 \%$ & 3 & $20.0 \%$ & & \\
\hline
\end{tabular}

$A C D F$ Anterior cervical discectomy and fusion, $D C I$ Dynamic cervical implant, SD Standard deviation, NS Non significant

\# Student $t$ test

${ }^{*}$ Chi-square tests

**Fisher's exact test

to the third and to the twelfth postoperative months, and a statistically significant value on comparing the improvement between the third and the twelfth months postoperatively (Table 2 ).

Similarly, a statistically highly significant improvement in VAS-A was noted on comparing the preoperative results to those at 1,3 , and 12 months postoperatively ( $P$ $=0.001$ ), and a highly significant value on comparing the first to the third and the twelfth postoperative months, while the comparison between the third to the twelfth postoperative months was statistically significant $(P=$ 0.041, Table 3).

Table 2 Comparison between preoperative VAS-N and at different follow-up intervals among ACDF group

\begin{tabular}{lll}
\hline & Mean & \pm SD \\
\hline Pre VAS-N & 5.13 & 1.302 \\
Post VAS-N 1 month & 1.73 & 0.704 \\
Post VAS-N 3 months & 0.87 & 0.743 \\
Post VAS-N 12 months & 0.53 & 0.640 \\
\hline
\end{tabular}

Repeated measure ANOVAs. Pre vs 1 month (HS), pre vs 3 months (HS), pre vs 12 months (HS), 1 month vs 3 months (HS), 1 month vs 12 months (HS), 3 months vs 12 months (S), using Post Hoc test

VAS-N Visual analogue scale for neck pain, SD Standard deviation, S Significant, HS Highly significant
Table 3 Comparison between preoperative VAS-A and at different follow-up intervals among ACDF group

\begin{tabular}{lll}
\hline & Mean & \pm SD \\
\hline Pre VAS-A & 7.87 & 0.743 \\
Post VAS-A 1 month & 1.20 & 0.862 \\
Post VAS-A 3 months & 0.60 & 0.632 \\
Post VAS-A 12 months & 0.33 & 0.488 \\
\hline
\end{tabular}

Repeated measure ANOVAs. Pre vs 1 month (HS), pre vs 3 months (HS), pre vs 12 months (HS), 1 month vs 3 months (HS), 1 month vs 12 months (HS), 3 months vs 12 months (S), using Post Hoc test

VAS-A Visual analogue scale for arm pain, SD Standard deviation, S Significant, HS Highly significant

The NDI improved postoperatively; the mean values revealed a statistically highly significant improvement on comparing the preoperative results to those at 1,3 , and 12 months postoperatively $(P=0.001)$, and a highly significant value on comparing the first to the third and to the twelfth postoperative months, while the comparison between the third to the twelfth postoperative months was statistically non-significant $(P>0.05$, Table 4$)$.

\section{$\mathrm{DCl}$ group}

Both VAS-N and VAS-A improved after DCI surgery; there was a statistically highly significant improvement regarding both VAS-N and VAS-A on comparing the preoperative to the postoperative results throughout the follow-up intervals $(P=0.001)$. Similarly, a highly significant value on comparing VAS-N of the first and the third postoperative months to the twelfth postoperative month $(P=0.001)$, in contrast to a statistically nonsignificant value $(P>0.05)$ on comparing the improvement between the first to the third month postoperatively (Table 5).

Also, a statistically highly significant value was noted on comparing VAS-A of the first to the third and to the twelfth postoperative months $(P=0.001)$, in contrast to a statistically non-significant value $(P>0.05)$ on comparing the improvement between the third to the twelfth months postoperatively (Table 6).

Table 4 Comparison between preoperative NDI and at different follow-up intervals among ACDF group

\begin{tabular}{lll}
\hline & Mean & \pm SD \\
\hline Pre NDI & 54.20 & 10.90 \\
Post NDI 1 month & 29.27 & 5.63 \\
Post NDI 3 months & 25.33 & 4.35 \\
Post NDI 12 months & 23.93 & 3.49 \\
\hline
\end{tabular}

Repeated measure ANOVAs. Pre vs 1 month (HS), pre vs 3 months (HS), pre vs 12 months (HS), 1 month vs 3 months (HS), 1 month vs 12 months (HS), 3 months vs. 12 months (NS), using Post Hoc test

NDI Neck disability index, SD Standard deviation, HS Highly significant, NS Non-significant 
Table 5 Comparison between preoperative VAS-N and at different follow-up intervals among DCI group

\begin{tabular}{lll}
\hline & Mean & \pm SD \\
\hline Pre VAS-N & 4.53 & 1.407 \\
Post VAS-N 1 month & 1.53 & 0.743 \\
Post VAS-N 3 months & 1.60 & 1.765 \\
Post VAS-N 12 months & 0.60 & 0.828
\end{tabular}

Repeated measure ANOVAs. Pre vs 1 month (HS), pre vs 3 months (HS), pre vs 12 months (HS), 1 month vs 3 months (NS), 1 month vs 12 months (HS), 3 months vs 12 months (HS), using Post Hoc test $V A S-N$ Visual analogue scale for neck pain, SD standard deviation, HS highly significant, NS non-significant

Compared to the preoperative data, the NDI score significantly decreased after surgery, the mean values revealed a statistically highly significant improvement on comparing the preoperative results to those at 1,3 , and 12 months postoperatively $(P=0.001)$, and a highly significant value on comparing the first and the third to the twelfth postoperative month; while the comparison between the first and the third postoperative months was statistically non-significant $(P>0.05$, Table 7$)$.

Comparing the $\mathrm{ACDF}$ and the DCI groups, there were no significant differences as regard baseline patient characteristics (Table 8). Also, there were no significant differences in clinical outcome at 1,3, and 12 months after surgery on comparing both groups regarding the VAS$\mathrm{N}$, VAS-A, and the NDI (Figs. 1, 2, and 3). However, subsidence was greater in the DCI group than it was in the ACDF group, showing a statistically significant value and a statistically highly significant value at 3 and 12 months, respectively $(P=0.035$ and $P=0.002$, Fig. 4). Similarly, the fusion rate was $80 \%$ in the ACDF group versus $26.7 \%$ the DCI group at 12 months postoperatively $(P=0.003)$. On the other hand, there was a statistically significant value $(P=0.011)$ on comparing the Cobb's angle mean values preoperatively and at 12 months postoperatively between both groups being higher in the ACDF group (13.79 \pm 3.10 and $16.28 \pm$ 3.17 , respectively) in contrast to the DCI group (14.07 \pm 3.474 and $15.453 \pm 3.8381$, respectively, Fig. 5).

Table 6 Comparison between preoperative VAS-A and at different follow-up intervals among DCI group

\begin{tabular}{lll}
\hline & Mean & \pm SD \\
\hline Pre VAS-A & 7.27 & 1.033 \\
Post VAS-A 1 month & 0.73 & 0.594 \\
Post VAS-A 3 months & 0.33 & 0.488 \\
Post VAS-A 12 months & 0.2 & 0.414 \\
\hline
\end{tabular}

Repeated measure ANOVAs. Pre vs 1 month (HS), pre vs 3 months (HS), pre vs 12 months (HS), 1 month vs 3 months (HS), 1 month vs 12 months (HS), 3 months vs. 12 months (NS), using Post Hoc test

VAS-A Visual analogue scale for arm pain, SD Standard deviation, HS Highly significant, NS Non-significant
Table 7 Comparison between preoperative NDI and at different follow-up intervals among DCl group

\begin{tabular}{lll}
\hline & Mean & \pm SD \\
\hline Pre NDI & 46.47 & 10.882 \\
Post NDI 1 month & 29.20 & 6.483 \\
Post NDI 3 months & 30.33 & 14.676 \\
Post NDI 12 months & 23.47 & 4.764 \\
\hline
\end{tabular}

Repeated measure ANOVAs. Pre vs 1 month (HS), pre vs 3 months (HS), pre vs 12 months (HS), 1 month vs 3 months (NS), 1 month vs 12 months (HS), 3 months vs. 12 months (HS), using Post Hoc test

$N D I$ Neck disability index, SD Standard deviation, HS Highly significant, NS Non-significant

Unintended durotomy occurred in a single patient in the ACDF group, the cerebrospinal fluid (CSF) leak was induced by a small dural tear that unfortunately was not accessible to allow a primary repair, consequently the tiny tear was covered intraoperatively with a small piece of Gelfoam (Pfizer, New York) augmented by a small autogenous fat graft then observed patiently, luckily complete cessation of CSF leakage was achieved; a sub muscular drain was kept in an non-dependant location with no suction while patient was confined to bed rest with his head elevated $30^{\circ}$ for two nights; then a stitch was taken at its place when removed on postoperative day 2; no subsequent collection or leakage was recorded. Implant migration was recorded in three $(20 \%)$ cases in the DCI group; all were presented between the fourth and the sixth week after the operation by acute onset of severe dysphagia and recurrent neck pain; plain radiographs revealed the migrating cage (Fig. 6), patients were reoperated, and a larger implant was introduced instead. Postoperatively, the patient experienced significant relief of the acute symptoms. No cage migration was recorded among the ACDF group.

A single patient of each group developed temporary recurrent laryngeal nerve injury on the same side of operation immediately after surgery; they received conservative medical management and had complete recovery within 1 month; similarly, one patient of each group had postoperative superficial wound infection that was successfully cured in less than 2 weeks using oral antibiotics and frequent wound dressings.

A single patient of the DCI group developed immediate postoperative $\mathrm{C} 5$ palsy (deltoid muscle paresis, motor Grade 3/5), yet there was no intraoperative neural injury to explain the unfortunate event, and the postoperative computed tomography (CT) scan did not reveal any surgical finding; patient subsequently had an intense physiotherapy and rehabilitation program and fully recovered deltoid function by the fourth postoperative month.

There was no statistically significant difference regarding the postoperative complications between the $\mathrm{DCI}$ 
Table 8 Comparison between the two study groups as regard clinical and radiological findings preoperatively and postoperatively

\begin{tabular}{|c|c|c|c|c|c|c|}
\hline & \multicolumn{4}{|c|}{ GROUP } & \multirow[t]{3}{*}{$P$} & \multirow[t]{3}{*}{ Significance } \\
\hline & \multicolumn{2}{|l|}{$\overline{\mathrm{ACDF}}$} & \multicolumn{2}{|l|}{$\mathrm{DCl}$} & & \\
\hline & Mean & $\pm \mathrm{SD}$ & Mean & $\pm \mathrm{SD}$ & & \\
\hline Cobb's angle pre & 13.79 & 3.10 & 14.07 & 3.47 & $0.822^{*}$ & NS \\
\hline Cobb's angle post 12 months & 16.29 & 3.17 & 15.45 & 3.84 & $0.522^{*}$ & NS \\
\hline Pre VAS-N & 5.13 & 1.30 & 4.53 & 1.41 & $0.236^{*}$ & NS \\
\hline Post VAS-N 1 month & 1.73 & 0.7 & 1.53 & 0.74 & $0.455^{*}$ & NS \\
\hline Post VAS-N 3 months & 0.87 & 0.74 & 1.6 & 1.76 & $0.149^{*}$ & NS \\
\hline Post VAS-N 12 months & 0.53 & 0.64 & 0.6 & 0.83 & $0.807^{*}$ & NS \\
\hline Pre VAS-A & 7.87 & 0.74 & 7.27 & 1.03 & $0.078^{*}$ & NS \\
\hline Post VAS-A 1 month & 1.20 & 0.86 & 0.73 & 0.59 & $0.095^{*}$ & NS \\
\hline Post VAS-A 3 months & 0.6 & 0.63 & 0.33 & 0.49 & $0.207^{*}$ & NS \\
\hline Post VAS-A 12 months & 0.33 & 0.49 & 0.2 & 0.41 & $0.426^{*}$ & NS \\
\hline Pre NDI & 54.20 & 10.90 & 46.47 & 10.88 & $0.062^{*}$ & NS \\
\hline Post NDI 1 month & 29.27 & 5.62 & 29.20 & 6.48 & $0.976^{*}$ & NS \\
\hline Post NDI 3 months & 25.33 & 4.35 & 30.33 & 14.68 & $0.223^{*}$ & NS \\
\hline Post NDI 12 months & 23.93 & 3.49 & 23.47 & 4.76 & $0.762^{*}$ & NS \\
\hline Subsidence 1 month (mm) & 5.65 & 0.50 & 5.59 & 0.49 & $0.742^{*}$ & NS \\
\hline Subsidence 3 months & 5.42 & 0.48 & 4.96 & 0.65 & $0.035^{*}$ & $S$ \\
\hline Subsidence 12 months & 5.33 & 0.45 & 4.49 & 0.79 & $0.002^{*}$ & $\mathrm{HS}$ \\
\hline \multicolumn{7}{|l|}{ Fusion at 12 months } \\
\hline No & 3 & $20.0 \%$ & 11 & $73.3 \%$ & $0.003^{* *}$ & $\mathrm{HS}$ \\
\hline Yes & 12 & $80.0 \%$ & 4 & $26.7 \%$ & & \\
\hline
\end{tabular}

*Student $t$ test

**Chi-square tests

$A C D F$ Anterior cervical discectomy and fusion, $D C I$ Dynamic cervical implant, VAS- $N$ Visual analogue scale for neck pain, VAS-A Visual analogue scale for arm pain, NDI Neck disability index, SD Standard deviation, NS Non-significant, S Significant, HS Highly significant

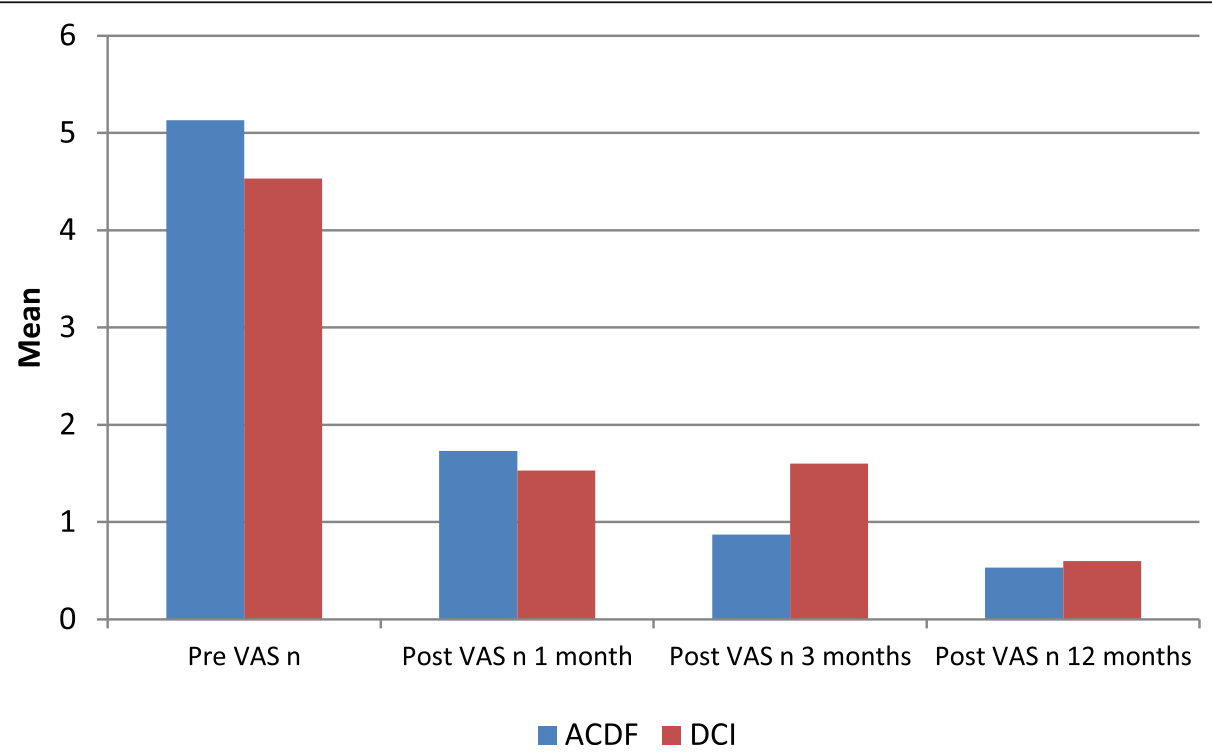

Fig. 1 Visual analogue score for the neck pain recorded preoperatively, and at 1, 3, and 12 months postoperatively for both groups 


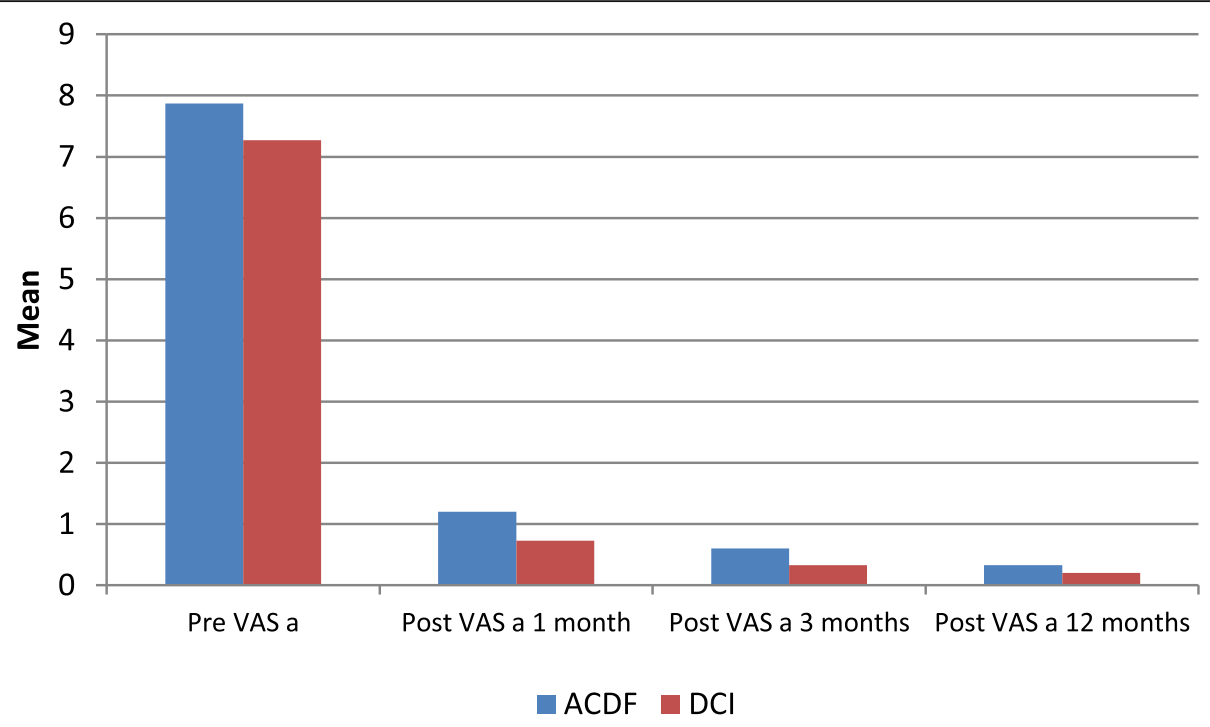

Fig. 2 Visual analogue score for the arm pain recorded preoperatively and at 1, 3, and 12 months postoperatively for both groups

group and the ACDF group (Table 9). Also, there was no statistically significant difference between both groups concerning operative time, intraoperative blood loss and length of hospital stay despite of showing shorter mean operative time and lesser intraoperative blood loss in the DCI group.

\section{Illustrative case}

This 30-year-old woman complained of neck pain and right brachialgia in a $\mathrm{C} 5$ radicular pattern for about 1 -year duration (VAS for neck and arm pain were recorded 4 and 6 , respectively, while her NDI was recorded 55); her pain was worse in extension; and conservative treatment was unsuccessful. Radiological evaluation revealed a $\mathrm{C} 4-5$ disc prolapse (Fig. 7). Subsequently, she was subjected to surgical decompression and dynamic stabilization with a DCI at C45.

These are the postoperative findings:

- Immediate postoperation, the patient's clinical outcome was excellent with good restoration of disc height, sagittal balance, and motion; right arm VAS improved to 2/10 and neck VAS was 2/10, while her NDI was 27; no operative complications took place.

- Postoperative plain X-rays at 1 month then at 12 months (Figs. 8 and 9, respectively) showed preserved cervical lordosis, maintained disc height, and

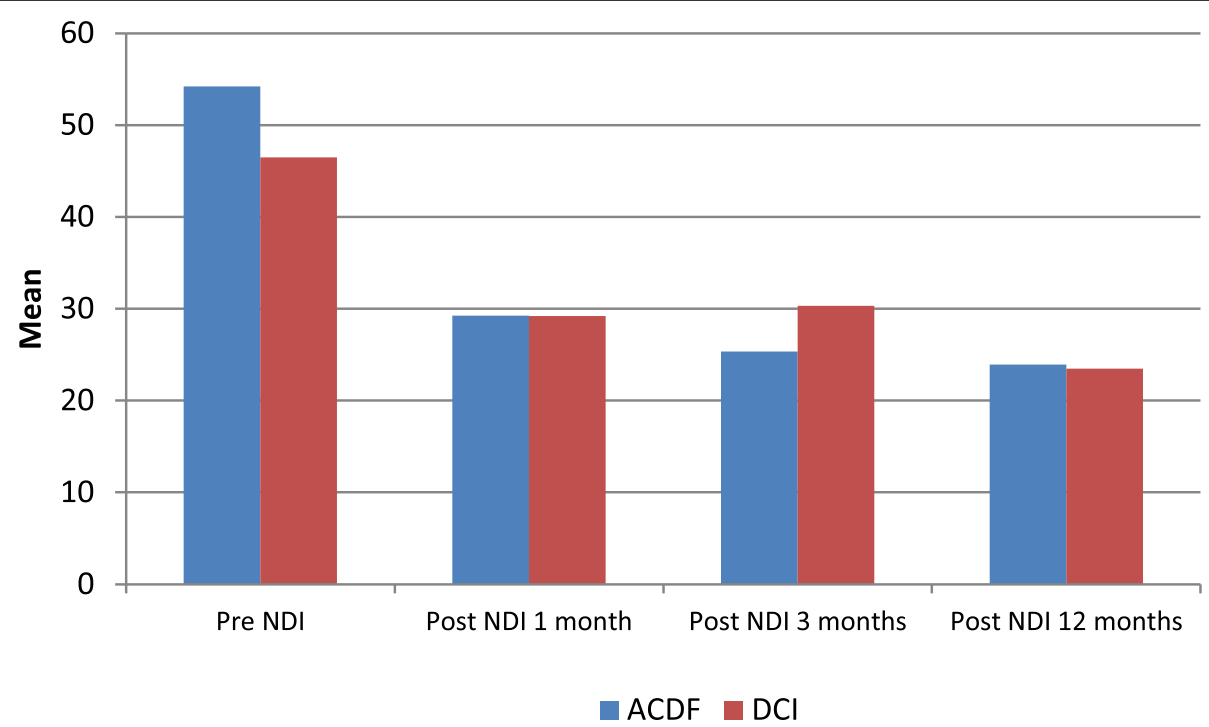

Fig. 3 Neck disability index recorded preoperatively and at 1, 3, and 12 months postoperatively for both groups 


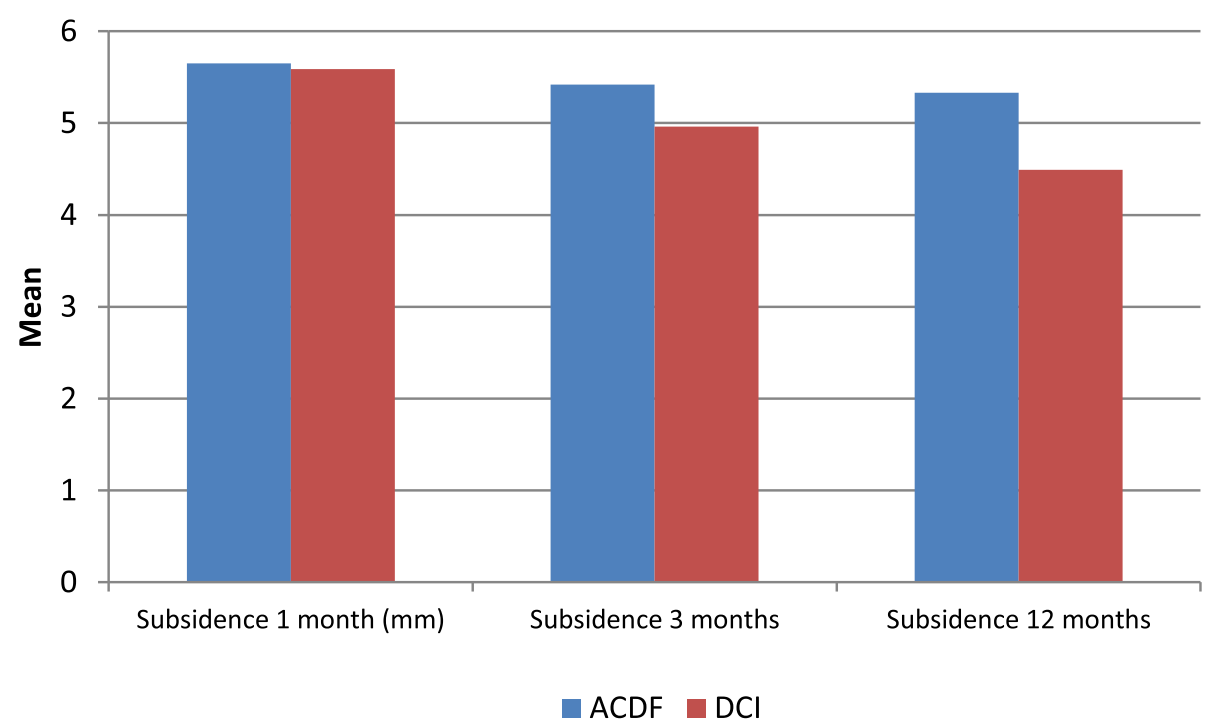

Fig. 4 Subsidence value at 1, 3, and 12 months postoperatively for both groups

segmental motion of the treated level with no evidence of fusion. One year postoperatively, both right arm and neck VAS showed further improvement to 1/10 each, while her NDI was 17.

\section{Discussion}

While ACDF provides excellent short-term outcomes, symptomatic adjacent segment degeneration may result following a process of increased loads and transfer of stresses to the adjacent discs, and consequently compromising their long-term health $[3,5]$; the DCI is considered a cervical implant philosophy aiming at providing cervical spine stability while preserving an adequate range of motion (ROM) at the involved segment without exacerbating excessive stress on the adjacent levels particularly in patients with pre-existing cervical DDD [9].

Our study was conducted on 30 patients having a singlelevel cervical disc prolapse presented with neck pain and radicular symptoms who were divided into the ACDF group $(n=15)$ and the DCI group $(n=15)$. Our initial results indicate that the DCI is able to maintain excellent neurological decompression and motion-segment stabilization. Clinical outcomes as evaluated by the VAS for neck and arm pain, and the NDI indicate that the DCI provides results comparable to ACDF.

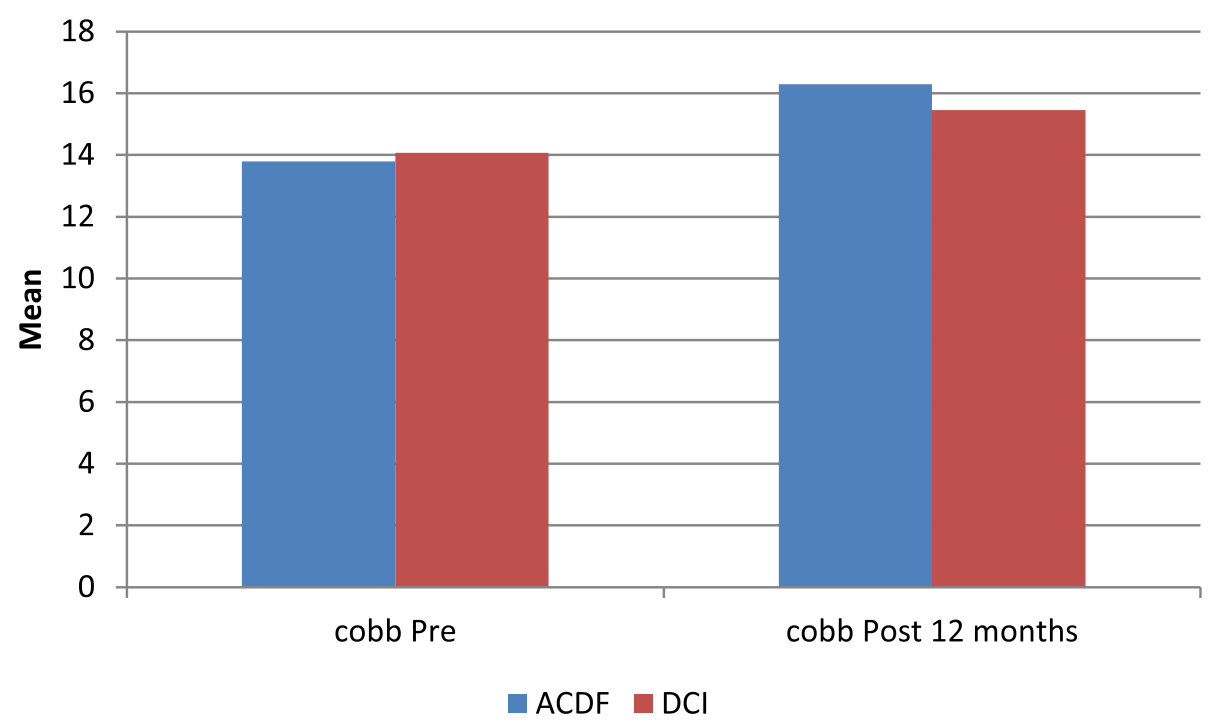

Fig. 5 Cobb's angle mean value preoperatively and at 12 months postoperatively for both groups 


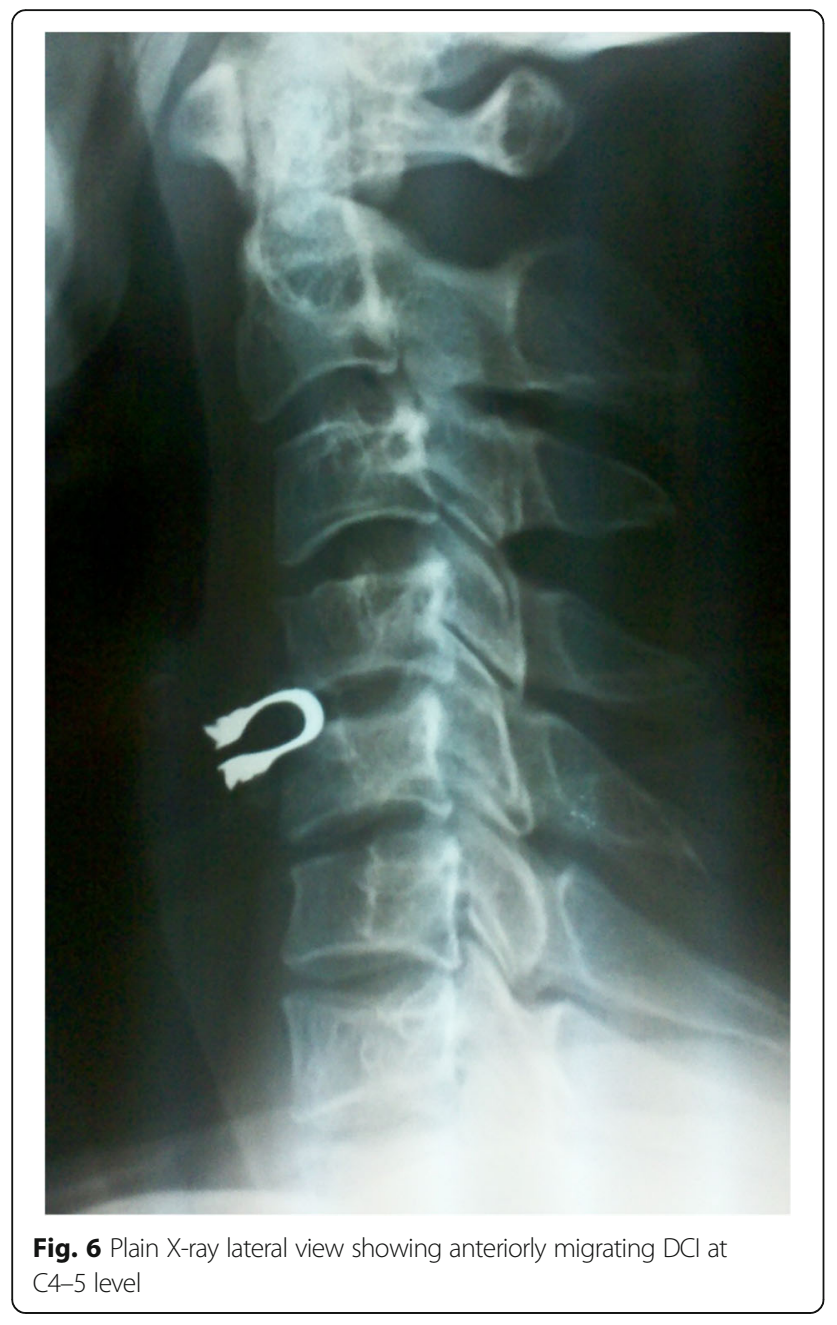

On comparing the preoperative to the postoperative clinical outcome in our study, both the DCI and the ACDF groups showed a significant improvement at 1, 3, and 12 months after surgery regarding the neck and arm pain, particularly over the first 3 postoperative months; these findings correspond with $\mathrm{Li}$ et al. [7] who reported the same significant improvements in clinical outcome measurements following both ACDF and DCI surgeries, with no significant differences between both groups.

In their retrospective comparative study, Zhu et al. [16] similarly compared both clinical and radiological outcome of ACDF versus DCI for the management of single-level cervical DDD, they reported that both groups showed significant clinical improvement showing comparable postoperative VAS and NDI score changes; however, the proportion of patients in the ACDF group who reported greater symptomatic satisfaction (described as very satisfied, or somewhat satisfied) was higher than that in the DCI group (95.3\% vs $79.1 \%)$. They declared that the long-term outcomes as a non-fusion technique were not that satisfactory; hence, they stopped using the DCI for 2 years following their study.

Wang et al. [15] presented the prospective results of 30 patients with single-level DDD managed by DCI. Similar to our results, they reported significant improvements in all clinical parameters postoperatively; however, they reported no implant dislocation, no spontaneous fusion, and no resorption at the bony end plate confirmed by computed tomography (CT) scans; all DCIs provided similar ROM pre and postoperatively in lateral plain X-ray in flexion and extension.

Concerning the radiological outcome 1 year postoperatively, our study revealed that subsidence was greater in the DCI group than it was in the ACDF group, showing a statistically highly significant value, a result similar to that obtained by Richter at al [13].. Since the DCI is a motion-preserving device, the rate of fusion is hence crucial. It was important to maximize the footprint of the implant and subsequently provide maximum coverage of the end plates, while the implant height was chosen by gently distracting the segment. Nevertheless, and in comparison to our study, Richter et al. described implant fusion in $39.4 \%$ of DCI cases (13 of 33 implants) at the twelfth month follow-up while our study noted a fusion rate of $26.7 \%$ at the same postoperative period in contrast to $80 \%$ in the ACDF group.

On the other hand, our study revealed a statistically significant difference on comparing the Cobb's angle mean values preoperatively and at 12 months postoperatively between both groups being higher in the ACDF group in contrast to the DCI group.

Li et al. [7] described clinical and radiologic outcomes following ACDF $(n=42)$ and DCI $(n=39)$ surgeries for single-level DDD over 2 years follow-up, and though describing similar clinical findings to our study as previously noted, however, they described an overall fusion rate of $94.9 \%$, considered probably in the ACDF group, whereas the fusion rate in the DCI group alone was not mentioned. Besides, they reported only two cases of implant subsidence in the DCI group (no criteria were noted), while our study revealed a significant decrease in the end-plate distance mean values from $5.59 \mathrm{~mm}$ to $4.49 \mathrm{~mm}$ on comparing results obtained at 1 month postoperatively versus 12 months follow-up radiographs among the DCI group, in contrast to $5.65 \mathrm{~mm}$ and 5.33 $\mathrm{mm}$ in the ACDF group, respectively. Moreover, fusion rate in the DCI group was not mentioned at all in their study.

Li et al. [7] reported a single case of DCI implant migration of $2 \mathrm{~mm}$ that appeared with no need for revision surgery, taking into consideration that $\mathrm{Li}$ et al. used 2$\mathrm{mm}$ dislocation as criteria. We had three cases of DCI anterior migration, all were presented between the fourth and the sixth week after the operation, they were 
Table 9 Perioperative data and operative complications

\begin{tabular}{|c|c|c|c|c|c|c|}
\hline & \multicolumn{4}{|l|}{ GROUP } & \multirow[t]{3}{*}{$P$} & \multirow[t]{3}{*}{ Sig } \\
\hline & \multicolumn{2}{|l|}{$\overline{\mathrm{ACDF}}$} & \multicolumn{2}{|l|}{$\mathrm{DCl}$} & & \\
\hline & Mean & $\pm S D$ & Mean & $\pm S D$ & & \\
\hline Operative time (min) & 90.67 & 17.41 & 85.67 & 17.82 & $0.443^{*}$ & $\overline{N S}$ \\
\hline Intra-operative blood loss (ml) & 115.33 & 22.95 & 108.67 & 23.56 & $0.439^{*}$ & NS \\
\hline Hospital stay (days) & 2.00 & 1.07 & 2.00 & 1.07 & $1.0^{*}$ & NS \\
\hline \multicolumn{7}{|l|}{ Cage ventral migration } \\
\hline No & 15 & $100.0 \%$ & 12 & $80.0 \%$ & \multirow[t]{2}{*}{$0.224^{* *}$} & \multirow[t]{2}{*}{ NS } \\
\hline Yes & 0 & $0.0 \%$ & 3 & $20.0 \%$ & & \\
\hline \multicolumn{7}{|l|}{ Dural tear } \\
\hline No & 14 & $93.3 \%$ & 15 & $100.0 \%$ & \multirow[t]{2}{*}{$1.0^{* *}$} & \multirow[t]{2}{*}{ NS } \\
\hline Yes & 1 & $6.7 \%$ & 0 & $.0 \%$ & & \\
\hline \multicolumn{7}{|l|}{ Superficial wound infection } \\
\hline No & 14 & $93.3 \%$ & 14 & $93.3 \%$ & \multirow[t]{2}{*}{$1.0^{* *}$} & \multirow[t]{2}{*}{ NS } \\
\hline Yes & 1 & $6.7 \%$ & 1 & $6.7 \%$ & & \\
\hline \multicolumn{7}{|l|}{ Rec. laryngeal N. injury } \\
\hline No & 14 & $93.3 \%$ & 14 & $93.3 \%$ & \multirow[t]{2}{*}{$1.0^{* *}$} & \multirow[t]{2}{*}{ NS } \\
\hline Yes & 1 & $6.7 \%$ & 1 & $6.7 \%$ & & \\
\hline \multicolumn{7}{|l|}{ C5 palsy (deltoid muscle paresis) } \\
\hline No & 15 & $100.0 \%$ & 14 & $93.3 \%$ & \multirow[t]{2}{*}{$1.0^{* *}$} & \multirow[t]{2}{*}{ NS } \\
\hline Yes & 0 & $0.0 \%$ & 1 & $6.7 \%$ & & \\
\hline
\end{tabular}

"Student $t$ test

**Fisher exact test

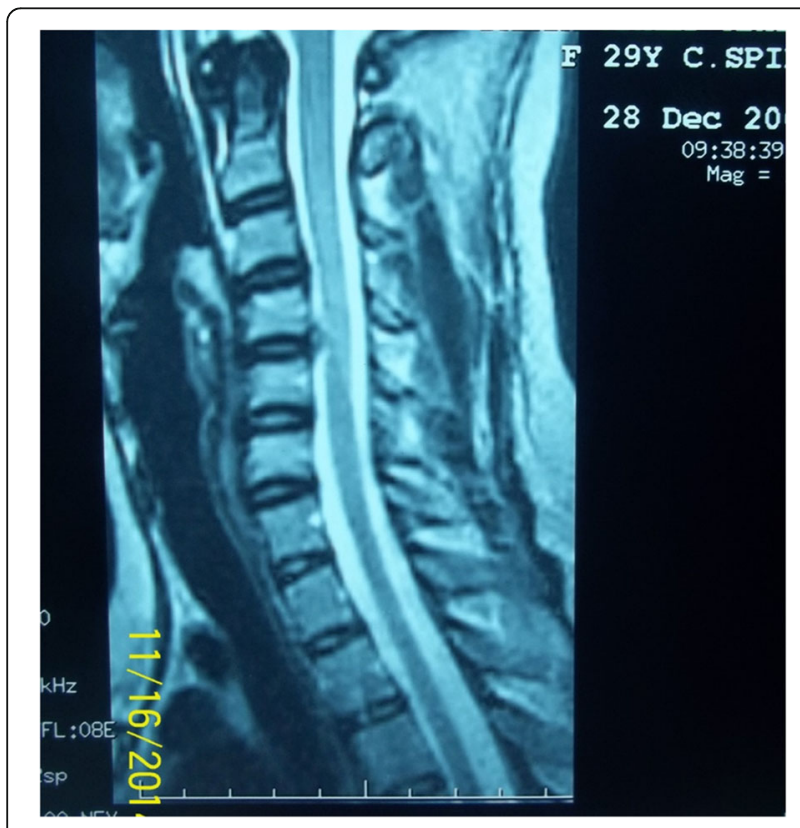

Fig. 7 Preoperative MRI sagittal T2W reoperated and a larger implant was introduced instead. Postoperatively, the patient experienced significant relief of the acute symptoms of recurrent neck pain and severe dysphagia.

Richter et al. [13] reported DCI migration in $15.2 \%$ of cases in comparison to $20 \%$ in our study, in contrast to the ACDF group where no cage migration was recorded in either studies. We consider this as a significant drawback of the DCI having a higher potential of migration and subsequent complications such as oesophageal injury, or recurrence of neck and/or radicular pain.

No remarkable complications were recorded in our study except a single case of unintended durotomy in the ACDF group, that was successfully managed conservatively; one patient of the DCI group experienced postoperative deltoid muscle paresis that was also managed conservatively and recovered completely 4 months following surgery; also, a single patient of each group developed immediate postoperative recurrent laryngeal nerve injury on the same side of the operation that was luckily a transient complication with a complete neurological recovery in 1 month; similarly, one patient of each group had superficial wound infection that was successfully cured in less than 2 weeks.

In summary, the incidence of postoperative complications was found statistically insignificant on comparing both groups of our study. 


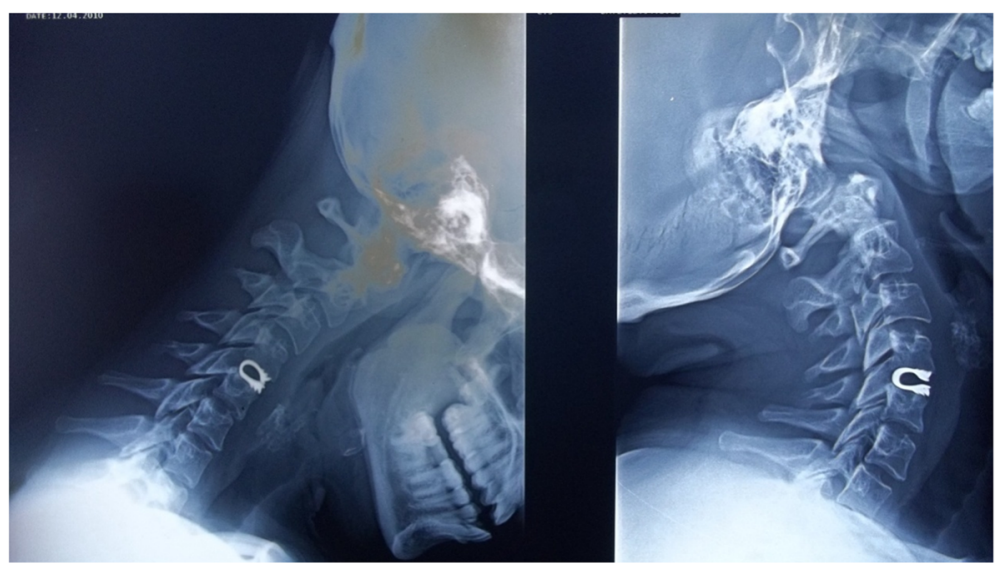

Fig. 8 One month postoperative dynamic cervical plain X-ray

Mohamed M. Mohi Eldin [11] reported a case of transient postoperative swallowing difficulty and another case with mild hoarseness of voice. Another case developed C6-7 spondylodiscitis, three levels away from the operated level (C3-C4) 2.5 months postoperatively. This case was managed conservatively and had resolution of symptoms within 3 months.

Zhu et al. [17] reported three cases in the ACDF group with postoperative dysphagia in the first week postoperatively, which recovered in 2 months.

Based on these results, we believe the main factor that contributes to considerable postoperative symptomatic relief is in fact based on a complete job of neural decompression rather than the type of the chosen implant.

Finally, the present study had several limitations, as the relatively small sample of subjects; also, a longer follow-up duration would still be required to assess the long-term clinical and radiological results.

\section{Conclusion}

Our study suggested that the DCI provides excellent clinical outcomes that appear similar to ACDF in the management of cervical disc disease. DCI presents a safe technique that provides satisfactory short-term results in treating single-level cervical disc prolapse in selected patients, without significant difference between the two groups regarding the operative time, intraoperative blood loss, complication rate, and length of hospital stay.

However, though providing an immediate dynamic stability, DCI is associated with higher rates of implant subsidence, lower fusion rates, and relatively high implant migration rate.

Also, there is no definitive evidence that DCI arthroplasty has better intermediate-term outcomes than ACDF with PEEK cage; consequently, further randomized controlled trials and long-term evaluation would be necessary to demonstrate the impact of this findings.

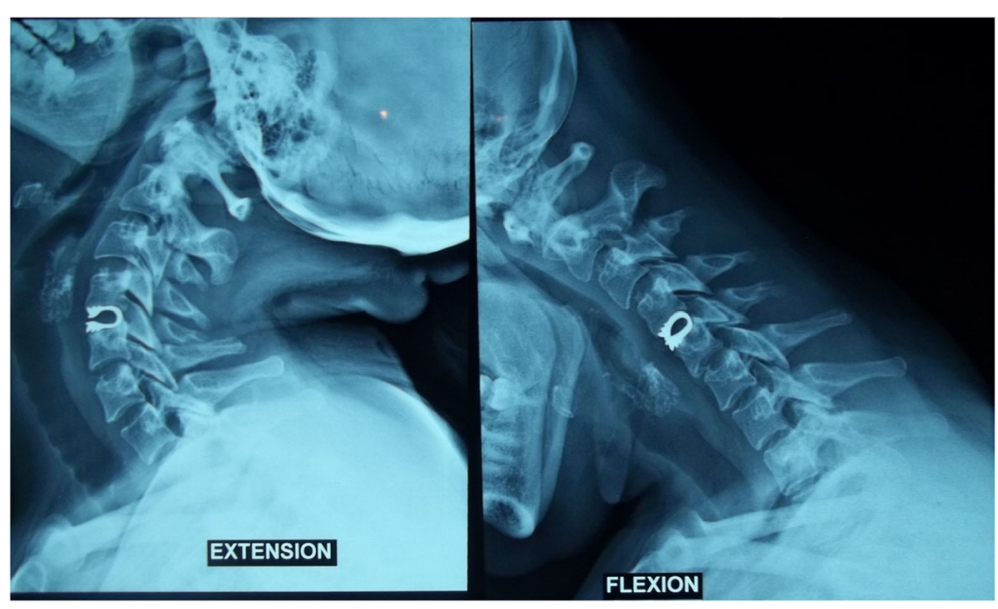

Fig. 9 One year postoperative dynamic cervical plain X-ray 


\section{Abbreviations}

ACDF: Anterior cervical discectomy and fusion; ANOVA: Analysis of variance; A-P: Anteroposterior; CSF: Cerebrospinal fluid; CT: Computed tomography; DCl: Dynamic cervical implant; DDD: Degenerative disc disease; HS: Highly significant; MRI: Magnetic resonance imaging; NDI: Neck disability index score; NS: Non-significant; PEEK: Polyethylethylketone; PXR: Plain X-ray; ROM: Range of motion; S: Significant; SD: Standard deviation; SPSS: Statistical Package for the Social Sciences; VAS-A: Visual analog scale for arm; VAS$\mathrm{N}$ : Visual analog scale for neck

\section{Acknowledgments}

Not applicable

\section{Authors' contributions}

The study design, execution and follow up of the clinical cases, data analysis and results formulation, and writing of the manuscript, were all the joint work of all the authors. All authors read and approved the final manuscript.

\section{Funding}

The authors received no external funding for the design of the study, for the collection, analysis, and interpretation of data or for writing the manuscript.

\section{Availability of data and materials}

All the raw data and results of the statistical analysis are available with the authors and ready to be shared with authorized personnel upon request; however, for reasons of patency protection it was not submitted with the manuscript.

\section{Ethics approval and consent to participate}

This research was conducted upon obtaining the approval of the ethical committee of the Faculty of Medicine, Ain Shams University, in July 2016. Since this study involved human subjects, an informed consent was signed and acquired from all the participants or their legal guardians in accordance with the ethical committee recommendations.

\section{Consent for publication}

Not applicable

\section{Competing interests}

The authors declare that they have no competing interests.

Received: 13 September 2019 Accepted: 13 January 2020

Published online: 19 February 2020

\section{References}

1. Baba H, Furusawa N, Imura S, Kawahara N, Tsuchiya H, Tomita K. Late radiographic findings after anterior cervical fusion for spondylotic myeloradiculopathy. Spine (Phila Pa 1976). 1993;18(15):2167-73.

2. Cunningham BW, Hu N, Zorn CM, McAfee PC. Comparative fixation methods of cervical disc arthroplasty versus conventional methods of anterior cervical arthrodesis: serration, teeth, keels, or screws? J Neurosurg Spine. 2010;12(2):214-20.

3. Hilibrand AS, Carlson GD, Palumbo MA, Jones PK, Bohlman HH. Radiculopathy and myelopathy at segments adjacent to the site of a previous anterior cervicalarthrodesis. J Bone Joint Surg Am. 1999;81(4): 519-28.

4. Huskisson EC. Measurement of pain. J Rheumatol. 1982;9(5):768-9.

5. Ishihara H, Kanamori M, Kawaguchi Y, Nakamura H, Kimura T. Adjacent segment disease after anterior cervical interbody fusion. Spine J. 2004;4(6): 624-8.

6. Lee CH, Hyun SJ, Kim MJ, Yeom JS, Kim WH, Kim KJ, et al. Comparative analysis of 3 different construct systems for single level anterior cervical discectomy and fusion: stand-alone cage, iliac graft plus plate augmentation, and cage plus plating. J Spinal Disord Tech. 2013;26(2):112-8,

7. Li Z, Yu S, Zhao Y, Hou S, Fu Q, Li F, et al. Clinical and radiologic comparison of dynamic cervical implant arthroplasty versus anterior cervical discectomy and fusion for the treatment of cervical degenerative disc disease. J Clin Neurosci. 2014;21(6):942-8.

8. Lin CY, Kang H, Rouleau JP, Hollister SJ, Marca FL. Stress analysis of the interface between cervical vertebrae end plates and the Bryan, Prestige LP, and ProDisc-C cervical disc prostheses: an in vivo image-based finite element study. Spine (Phila Pa 1976). 2009:34(15):1554-60.

9. Matgé G, Berthold C, Gunness VR, Hana A, Hertel F. Stabilization with the dynamic cervical implant: a novel treatment approach following cervical discectomy and decompression. J Neurosurg Spine. 2015;22(3):237-45.

10. Matgé $G$, Buddenberg P, Eif M, Schenke H, Herdmann J. Dynamic cervical stabilization: a multicenter study. Eur Spine J. 2015;24(12):2841-7.

11. Mohi Eldin MM. Dynamic cervical implant (DCI) in single level cervical disc disease.Open. Spine J. 2014;6:1-8

12. Papadopoulos EC, Huang RC, Girardi FP, Synnott K, Cammisa FP Jr. Threelevel anterior cervical discectomy and fusion with plate fixation: radiographic and clinical results. Spine (Phila Pa 1976). 2006;31(8):897-902.

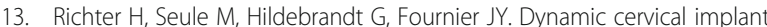
versus anterior cervical diskectomy and fusion: a prospective study of clinical and radiologic outcome. J Neurol Surg A Cent Eur Neurosurg. 2016; 77(4):300-7.

14. Vernon $\mathrm{H}$, Mior $\mathrm{S}$. The neck disability index: a study of reliability and validity. J Manipulative Physiol Ther. 1991;14(7):409-15.

15. Wang L, Song YM, Liu LM, Liu H, Li T. Clinical and radiographic outcomes of dynamic cervical implant replacement for treatment of single-level degenerative cervical disc disease: a 24-month follow-up. Eur Spine J. 2014; 23(8):1680-7.

16. Zhu C, Yang X, Wang L, Hu BW, Liu LM, Wang LN, et al. Comparison of dynamic cervical implant versus anterior cervical discectomy and fusion for the treatment of single-level cervical degenerative disc disease: a five-year follow-up. Clin Neurol Neurosurg. 2018;164:103-7.

17. Zhu R, Yang H, Wang Z, Wang G, Shen M, Yuan Q. Comparisons of three anterior cervical surgeries in treating cervical spondyloticmyelopathy. BMC Musculoskelet Disord. 2014;15:233.

\section{Publisher's Note}

Springer Nature remains neutral with regard to jurisdictional claims in published maps and institutional affiliations.

\section{Submit your manuscript to a SpringerOpen ${ }^{\circ}$ journal and benefit from:}

- Convenient online submission

- Rigorous peer review

- Open access: articles freely available online

High visibility within the field

- Retaining the copyright to your article

Submit your next manuscript at $\boldsymbol{\nabla}$ springeropen.com 